\title{
An Acute Leukemia Case with B/T-ALL Phenotypes
}

\section{Osman Yokuş ${ }^{1 *}$ and Habip Gedilk2*}

${ }^{1}$ Hematologist and internal diseases physician, Department of Hematology, Ministry of Health İstanbul Training and Research Hospital, İstanbul

${ }^{2}$ Infectious diseases and clinical microbiology physician, Department of infectious diseases and clinical microbiology, Ministry of Health Bakırköy Sadi Konuk Training and Research Hospital, İstanbul

*Corresponding author: Habip Gedik, Department of infectious diseases and clinical microbiology, Bakırköy Sadi Guest Education and Research Hospital, Istanbul-Turkey, Tel: +90 21231455 55; Fax: +90 21222178 00; E-mail: habipgedik@yahoo.com

Received date: October 05, 2015; Accepted date: December 01, 2015; Published date: December 10, 2015

Copyright: (C) 2015 Gedik H, et al. This is an open-access article distributed under the terms of the Creative Commons Attribution License, which permits unrestricted use, distribution, and reproduction in any medium, provided the original author and source are credited.

\section{Abstract}

Biphenotypic acute leukemia is defined as acute undifferentiated leukemia that does not have a single lineage specific antigen and expresses antigens that belong to multiple different lineages in varying degrees. In this study, a case with biphenotypic leukemia is being presented in terms of clinical course and prognosis. As a result, biphenotypic leukemia has different biological characteristics in terms of both immunophenotypic and cytogenetic characteristics.

Keywords: Leukemia; Biphenotypic; Acute myeloid leukemia

\section{Introduction}

Acute leukemia $(A L)$, which originates from lymphoid or myeloid stem cells, is classified as acute lymphoblastic leukemia (ALL) or acute myeloid leukemia (AML) according to morphological, cytochemical, and immunophenotypic features of the blast. Ambiguous lineage acute leukemia, which comprises of less than $4 \%$ of all leukemias, does not belong to a single lineage according to WHO classification in 2008 [1]. Biphenotypic acute leukemia is defined as acute undifferentiated leukemia that does not have a single lineagespecific antigen and expresses antigens that belong to multiple different lineages in varying degrees [1]. They could be seen as each blast of two different blast population expressing antigen belonging to different lineage that was formerly called bilineage leukemia, or antigen expression belonging to multiple lineages in the single blast population that is called as biphenotypic acute leukemia [1]. Complex karyotype anomalies, such as $t$ (9:22), gene MLL (mixed lineage leukemia gene) are common in those leukemias. Mixed phenotype acute leukemias (MPAL) are rare and its incidence that was reported to be less than $2 \%$ of all leukemias according to WHO classification in 2008 [2].

In this study, a case with biphenotypic leukemia is being presented in terms of clinical course and prognosis.

\section{Case}

A 25-year old male case was admitted with fever, night sweat, and weight loss. Blood smear was performed as cytopenia was in complete blood count (CBC). Bone marrow biopsy was performed as atypical blast was seen in blood smears. Biphenotypic leukemia (B-ALL/T-ALL) was diagnosed with bone marrow biopsy and flow cytometry. Abnormal findings were white blood cell (WBC): 2,400 cells $/ \mathrm{mm}^{3}$, hematocrit: $24 \%$, platelet: 24,700 cells $/ \mathrm{mm}^{3}$ in CBC; lactate dehydrogenase (LDH): $1382 \mathrm{U} / \mathrm{L}$ and INR: 1.2 in biochemistry analysis; hepatosplenomegaly and multiple mesenteric lymph adenomegaly (the largest one was measured in size with $1.5 \times$ $1 \mathrm{~cm}$ ) in abdomen ultrasonography. Flow cytometry resulted as cCD3: $73 \%$, CD7: 75\%, CD34: 51\%, CD22: 15\%, cCD79a: 58.8\%, CD22: 22\%, CD38: $72.5 \%$, CD33: $29 \%$, TdT: $57.3 \%$. The findings were compatible with ALL showing aberrant CD79a expression compatible with the european group for the immunological classification of leukemias (EGIL) T-I in Tlymphoid series, but it was less likely to be biphenotypic leukemia, including $T$ and $B$ lymphoid origins (T-ALL was predominating, but there were $T$ and B ALL markers that were considered as biphenotypic leukemia). Conventional cytogenetic analysis revealed complex karyotype abnormalities, including 44-48, $X Y,+Y\{2\}$, t (6:11) (q12; p14) $\{7\},+8\{3\},+19\{6\},\{c p 7\} / 46, X Y\{13\}$. Hyper-CVAD $1 A$ regimen was administered. During febrile neutropenia episode, Meropenem, Tazobactam-Piperacillin, Acyclovir, Caspofungin, Trimethoprim-Sulfamethoxazole and Vancomycin were administered. On day 28, bone marrow aspiration and biopsy were performed for remission control (Figure 1). 


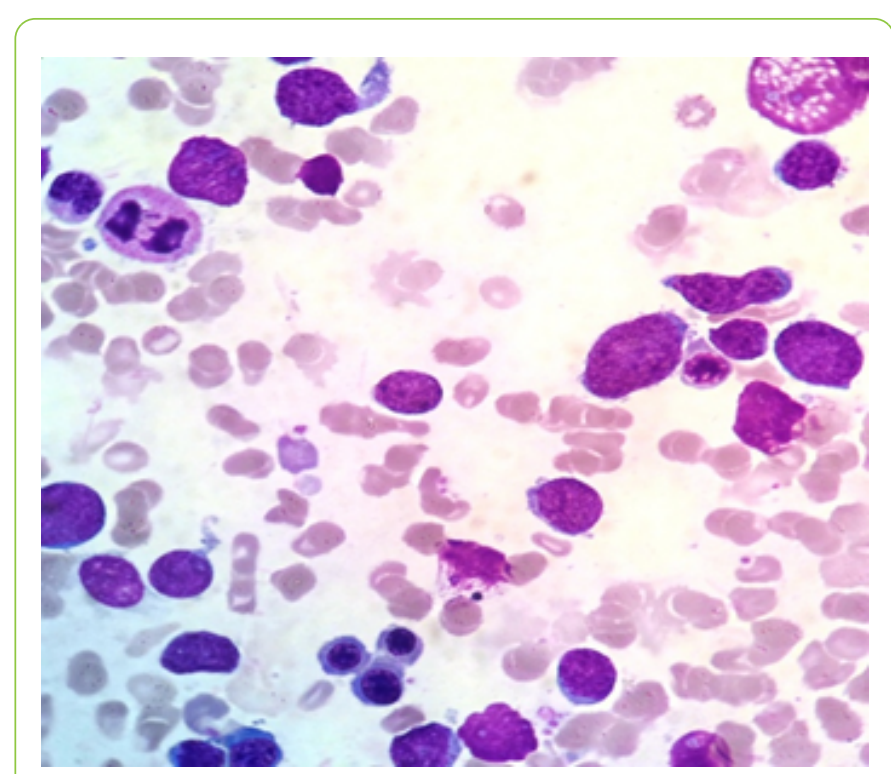

Figure 1: Atypical blast cells (80\%) in the bone marrow biopsy after Hyper CVAD-1A chemotherapy (Giemsa: $1 \times$ 100).

Bone marrow aspirate smears revealed two different sizes (small and large) blast population at the rate of eighty percent and suppression in other series. In addition, flow cytometry results of bone marrow were not consistent with remission. He had the left upper quadrant pain referred in left shoulder and relieved with Tradamol. His pain increased and the long diameter of splenomegaly was measured as $230 \mathrm{~mm}$. Palliative radiotherapy was administered to spleen and then HyperCVAD 1B regimen was administered On day 25 , bone marrow aspiration and biopsy control revealed atypical blast cells that some cells had either double or triple nucleus (Figure 2).

Salvage FLAG+IDA regimen was administered, but treatment response was not achieved once again (Figure 3 ). The patient died of sepsis.

\section{Discussion}

A small subset of acute leukemia, which has both myeloid and lymphoid lineage markers, is defined as mixed lineage or biphenotypic acute leukemia. Biphenotypic acute leukemia (BAL) is quite rare and composes $2 \%$ of all acute leukemias originating from common lymphoid progenitor cell $[3,4]$. Leukemias are generally defined according to the european group for the immunological classification of leukemia (EGIL). This system is based on the number (marker) and specificity (lymphoid or myeloid antigen) of aberrant antigen expression in the blasts. Biphenotypic acute leukemia (BAL) often originates from multipotent progenitor cells and has a poor prognosis. It is unknown exactly treatment is administered targeting AML or ALL. High-dose chemotherapy and stem cell transplantation are recommended for complete eradication of the disease. Blasts are seen as AML or ALL (often in the AMLM1 type), and both rarely can be seen together as it was seen in our case. Some blasts can be seen either big or small (like lymphoblasts) in a single area of blood smear [5].

A study evaluating clinical and biological characteristics of 22 biphenotypic acute leukemia cases reported that they were older age compared to adult ALL cases, and resistant to chemotherapy (response rates of $65 \%$ in BAL; $86 \%$ in ALL; $\mathrm{P}=0.04$ ), and cytogenetic analysis resulted abnormal karyotype in $56 \%$ of cases. Distinguishing features of BAL were to be seen in elderly, more expression of the CD34 antigen, to have often Philadelphia chromosome, and to be observed spleen, liver, and lymph node infiltration [6]. Biphenotypic acute leukemia is a high-risk acute leukemia and more resistant to chemotherapy than ALL and ALL due to underlying clonal abnormalities; therefore, it should be treated with more intensive chemotherapy. There is no a definitive treatment yet, but treatment recommendation depends on immunophenotyping and gene rearrangement profile [7]. Clonal chromosomal abnormalities were observed in 59-90\% of the cases [8]. The complex karyotype anomaly, which is associated with poor prognosis, was seen in our case. Unresponsiveness to hyper-CVAD $1 \mathrm{~A}$ and $1 \mathrm{~B}$ and then salvage FLAG-IDA chemotherapies, painful massive splenomegaly that was relived with tramadol and needed radiotherapy suggested poor prognosis in our case as well.

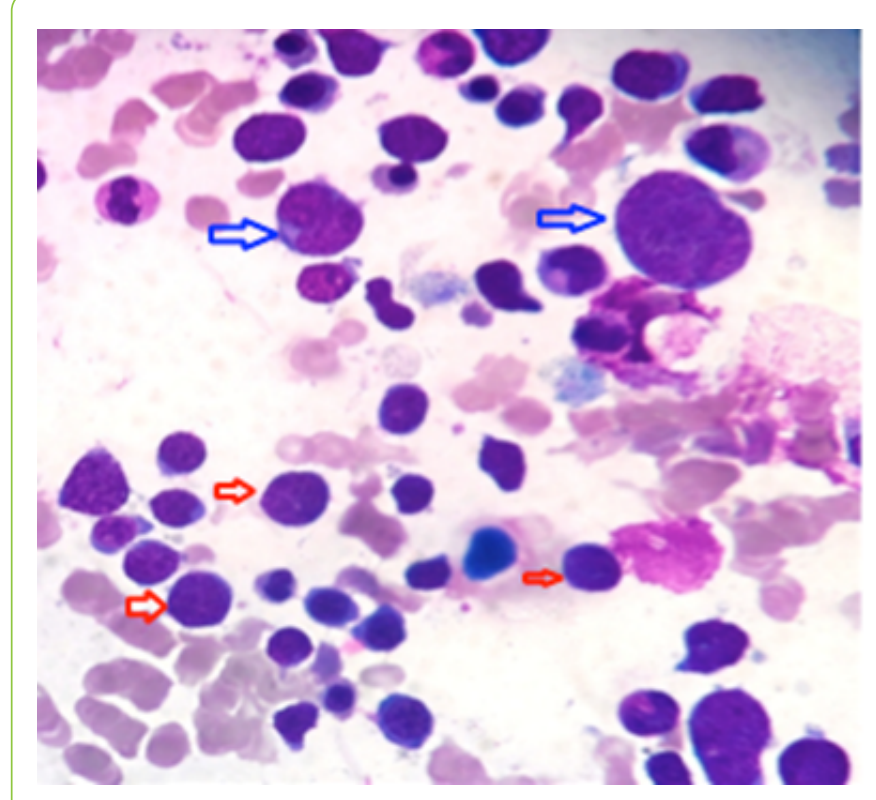

Figure 2: Two different blast cells in the bone marrow preparations (Red arrows point small blast cells; blue arrows point big blast cells, Giemsa: $1 \times 100$ ).

In a study evaluating the immunophenotypic features of 45 patients diagnosed with biphenotypic acute leukemia; cCD79a and cMPO followed by high level CD19 and CD10 expressions; CCD3 and CMPO followed by CD7 and CD5 expression in 18 cases with My/T-ALL; cCD3 and CCD79a co-expression in four cases with T/B-ALL were determined. TdT expression in 30 cases, CD34 expressions in 25 cases, CD117 expression in 31 cases was found as well. CD33 is more expressed than CD13 antigen in My/B-ALL and My/T-ALL patients. Treatment 
response rates were reported to be higher in cases with My/BALL CD34 (-) than cases with My/B-ALL ile My/T-ALL CD34 (+), and in cases with My/T-ALL CD34 (-) than cases with My/B-ALL CD34 (+). The Co-expression is more observed in My/B-ALL than My/T-ALL. Antigens including CCD3, cCD79a, cMPO are determined in for diagnosis of BAL. The four-color direct immunofluorescence staining with flow cytometry was reported to be very specific for diagnosis of BAL.

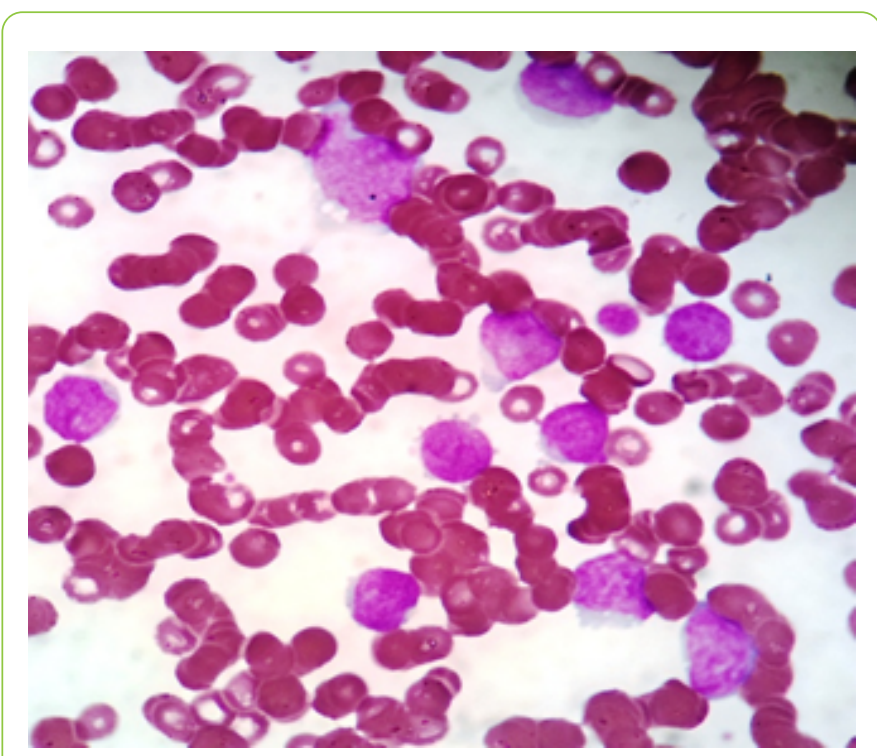

Figure 3: Blast cells after salvage FLAG+IDA chemotherapy in the blood smear.

The most common cytogenetic alterations in mixed phenotypic acute leukemia are $t(9,22)$ (q34;q11) BCR-ABL1, and 11q23 mixed lineage leukemia rearrangement. Using the database of the Surveillance, Epidemiology, and End Results registry (SEER), 313 patients with mixed phenotype acute leukemias were identified and compared them with 14,739 patients with acute lymphoblastic leukemia and 34,326 patients with acute myelogenous leukemias diagnosed between 2001 and 2011. The incidence of mixed phenotype acute leukemias was reported to be 0.35 cases $/ 1,000,000$ person-years. In a multivariate analysis, the prognosis was reported to depend strongly on age (as with other leukemias) and it has the worst outcome of all four types of leukemia.

As a result, biphenotypic leukemia has different biological characteristics in terms of both immunophenotypic and cytogenetic characteristics. Subgroup of T/B-ALL leukemia is rarely seen and has the worst prognosis with mixed phenotype.

\section{Conflict of Interest}

The author has no conflict of interest.

\section{References}

1. Lau LG, Tan LK, Koay ES, Ee MH, Tan SH, et al. (2004) Acute lymphoblastic leukemia with the phenotype of a putative Bcell/T-cell bipotential precursor. Am J Hematol 77: 156-160.

2. Manola KN (2013) Cytogenetic abnormalities in acute leukaemia of ambiguous lineage: an overview. Br J Haematol 163: 24-39.

3. Weinberg OK, Arber DA (2010) Mixed-phenotype acute leukemia: historical overview and a new definition. Leukemia 24: 1844-1851.

4. Matutes E, Morilla R, Farahat N, Carbonell F, Swansbury J, et al. (1997) Definition of Acute biphenotypic leukemia. Haematologica 82: 64-66.

5. Tong H, Liu Z, Lu C, Wang Q (2013) Clinical and laboratory features of adult biphenotypic acute leukemia. Asia Pac J Clin Oncol 9: 146-154.

6. Kaćanski N, Konstantinidis N, Kolarović J, Slavković B, Vujić D (2010) Biphenotypic acute leukaemia: case reports of two paediatric patients. Med Pregl 63: 867-869.

7. Zhang JJ, Zhang N, Zhang XF, Shi Y, Wang H (2011) Immunophenotyping characteristics of biphenotypic acute leukemia and analysis of curative effect. Zhongguo Shi Yan Xue Ye Xue Za Zhi 19: 317-320.

8. Shi R, Munker R (2015) Survival of patients with mixed phenotype acute leukemias: a large population-based study. Leuk Res 39: 606-616. 